\title{
VESTIBOLOGY
}

\section{Evolution of migraine vertigo in overlapping syndrome with Ménière's disease: prognostic role of instrumental examination}

\author{
Evoluzione dell'emicrania vestibolare in sindrome da sovrapposizione con la malattia \\ di Ménière: ruolo prognostico degli esami strumentali
}

\author{
Francesco Dispenza ${ }^{1,2}$, Angelo Immordino ${ }^{3}$, Roberta Priola ${ }^{3}$, Pietro Salvago ${ }^{3}$, Calogero Montalbano ${ }^{3}$, Francesco Martines ${ }^{2,3}$ \\ ${ }^{1}$ U.O.C. Otorinolaringoiatria, A.U.O. Policlinico P. Giaccone, Palermo, Italy; ${ }^{2}$ Istituto Euro-Mediterraneo di Scienza e Tecnologia - \\ IEMEST, Palermo, Italy; ${ }^{3}$ Dipartimento di Biomedicina, Neuroscienze e Diagnostica Avanzata, Università degli Studi di Palermo, Italy
}

\begin{abstract}
SUMMARY
Introduction. Migraine vertigo (MV) and Ménière's disease (MD) share several signs and symptoms such as tinnitus, fullness, photophobia, phonophobia, headache and vertigo spells lasting hours.

Objective. The aim of the present study was to prospectively observe patients with MV with a sporadic audiological symptom evaluated with clinical examination, Vestibular Evoked Myogenic Potentials (VEMPs), ECochG and v-HIT.

Results. The finding of VEMP asymmetry, according to our cut-off of $33 \%$ of difference between sides, resulted in 20 cases, of which 6 had asymmetry of both c-VEMPs and oVEMPS, all with development of fluctuating hearing during follow-up. ECochG was positive for endolymphatic hydrops in 12 patients.

Conclusions. The evolution of MV may have a variable course in which some patients may develop symptoms typical of MD. The two diseases may be contextually present at the same time configuring an overlapping syndrome, and asymmetric VEMPs might predict development of fluctuating hearing.
\end{abstract}

KEY WORDS: vestibular migraine, Ménière's disease, overlapping syndrome, vertigo, VEMPs, ECochG

\section{RIASSUNTO}

Introduzione. La vertigine emicranica e la malattia di Ménière condividono diversi segni e sintomi come acufeni, ovattamento auricolare, fonofobia, cefalea e crisi di vertigine della durata di ore.

Obiettivo. Lo scopo dello studio è stato quello di valutare prospetticamente i pazienti con vertigine emicranica e manifestazioni audiologiche sporadiche, mediante esame clinico, VEMPs, ECOchG, v-HIT.

Risultati. Il riscontro di asimmetria dei VEMPs, secondo il limite del 33\% di differenza tra i due lati, si è avuto in 20 casi dei quali: 6 con asimmetria di ocular e cervical VEMPs e sviluppo di fluttuazione uditiva nel corso del follow-up. L'ECochG ha riscontrato un'idrope endolinfatica in 12 pazienti.

Conclusioni. L'evoluzione della vertigine emicranica durante la vita del paziente può avere un decorso variabile nel quale alcuni di essi possono sviluppare sintomi tipici della malattia di Meniere. Le due malattie possono essere contestualmente presenti configurando la sindrome da sovrapposizione e l'asimmetria di entrambi $i$ VEMPs potrebbe predire lo sviluppo della fluttuazione uditiva.

PAROLE CHIAVE: emicrania vestibolare, malattia di Ménière, sindrome da sovrapposizione, vertigine, VEMPs, elettrococleografia

\section{Introduction}

Migraine vertigo (MV) and Ménière's disease (MD) share several signs and symptoms such as tinnitus, fullness, photophobia, phonophobia, headache and vertigo spells lasting hours ${ }^{1,2}$. Diagnosis of MD or MV is based on anamnes-
Received: March 18, 2020

Accepted: October 31, 2020

Correspondence

Francesco Dispenza

via Antonio di Rudinì 24/C, 90123 Palermo, Italy

E-mail: francesco.dispenza@gmail.com

Funding

None.

Conflict of interest

The Authors declare no conflict of interest.

How to cite this article: Dispenza F, Immordino A, Priola R, et al. Evolution of migraine vertigo in overlapping syndrome with Ménière's disease: prognostic role of instrumental examination. Acta Otorhinolaryngol Ital 2021;41:180-184. https:// doi.org/10.14639/0392-100X-N0752

(c) Società Italiana di Otorinolaringoiatria e Chirurgia Cervico-Facciale

\section{c) (1) $(9)$}

This is an open access article distributed in accordance with the CC-BY-NC-ND (Creative Commons Attribution-NonCommercial-NoDerivatives 4.0 International) license. The article can be used by giving appropriate credit and mentioning the license, but only for non-commercial purposes and only in the original version. For further information: https:// creativecommons.org/licenses/by-nc-nd/4.0/deed.en 
tic data and clinical features, making differential diagnosis very difficult ${ }^{3}$.

In 2013, the International Headache Society published the last classification of headache diseases ICHD3-beta including the up-to-date diagnostic criteria of $\mathrm{MV}^{4}$, and in 2015 the diagnostic criteria for MD are codified by the European Academy of Otology and Neurotology ${ }^{5}$; both classifications are used widely to diagnose these two diseases (Tab. I).

However, in clinical practice, several patients exhibit features of both diseases. Murofushi et al. defined such patients as suffering from an overlapping syndrome of $\mathrm{MD}$ and $\mathrm{MV}^{6}$. The aim of the present study was to prospectively observe a series of patients with definite MV having some sporadic audiological symptom (i.e. tinnitus, fullness), but normal hearing, and followed-up with electrophysiological and clinical tests. All instrumental findings were then correlated with clinical behaviour.

\section{Materials and methods}

Among all patients with vestibular diseases who referred to our Laboratory of Acoustic Electrophysiology and Vestibology in 2016, we prospectively evaluated patients matching criteria for MV reported by ICHD3-beta and Barany Society. All patients had focused clinical history: number of severe vertigo spells, dizziness evaluated with Dizziness Handicap Inventory Italian validated version ${ }^{7}$, subjective tinnitus, fullness, subjective hearing loss and fluctuating hearing, aura symptom, triggers for headache and vertigo, anxiety, motion sickness and ascertained food intolerance.
Otomicroscopy and pure tone audiometry (PTA) testing bone and air conduction for $0.25-0.5-1-2-3-4 \mathrm{kHz}$ was done in all patients. All patients also underwent tympanometry and stapedial reflexes study.

The exclusion criteria were: previous diagnosis of MD, vestibular schwannoma of VIII cranial nerve, benign paroxysmal positional vertigo, head trauma, middle ear diseases, conductive hearing loss (i.e. suspect for otosclerosis), sensorineural hearing loss, labyrinthine fistula, ototoxic drug exposure, barotrauma, middle and inner ear malformations, chronic neuropathy (i.e. diabetes, demyelinating disease, chronic renal failure) and alteration of stapedial reflex or type B and C tympanogram.

Clinical vestibular examination included: searching for spontaneous nystagmus with video-Frenzel, Dix-Hallpike test and supine roll test to exclude positional vertigo, head shaking test, head impulse test, skew test and Romberg test. Cervical and Ocular VEMPs (c-VEMPs and o-VEMPs) were recorded in all patients with Socrates, Hedera Biomedics S.r.l. with biofeedback of muscle contraction on the monitor. Biofeedback is used to reduce the variability in case of difference in muscle contraction between sides. It is achieved through a digital LED and displayed waveform rectification, for a realistic comparison between the ipsiand contralateral amplitudes.

Regarding o-VEMPs, the negative electrode was positioned under the eye contralateral to the acoustic stimulation side and the positive electrode a few mm under the negative one, with the ground electrode on the forehead. The patient was in a supine position and the recording started by asking pa-

Table I. Diagnostic criteria for vestibular migraine and Ménière's disease according to the last available classification.

International classification headache disease 3-beta: vestibular migraine diagnostic criteria

\section{Vestibular migraine}

A. At least 5 episodes with vestibular symptoms of moderate or severe intensity, lasting 5 minutes to 72 hours

B. Current or previous history of migraine with or without aura according to the ICHD

C. One or more migraine features with at least $50 \%$ of the vestibular episodes: Headache with at least two of the following characteristics: one sided location, ear pulsating quality, moderate or severe pain intensity, aggravation by routine physical activity

Photophobia and phonophobia

Visual aura

D. Not better accounted for by another vestibular or ICHD diagnosis

\section{Probable vestibular migraine}

A. At least 5 episodes with vestibular symptoms of moderate or severe intensity, lasting 5 minutes to 72 hours

B. Only one of the criteria $B$ and $C$ for vestibular migraine is fulfilled (migraine history or migraine features during the episode)

C. Not better accounted for by another vestibular or ICHD diagnosis
European Academy Otology Neurotology Ménière's disease diagnostic criteria

\section{Definite Ménière's disease}

A. Two or more spontaneous episodes of vertigo, each lasting 20 minutes to 12 hours

B. Audiometrically documented low- to medium-frequency sensorineural hearing loss in the affected ear on at least one occasion before, during or after one episodes of vertigo

C. Fluctuating aural symptoms (hearing, tinnitus or fullness) in the affected ear

D Not better accounted for by another vestibular diagnosis

\section{Probable Ménière's disease}

A. Two or more episodes of vertigo or dizziness, each lasting 20 minutes to 24 hours

B. Fluctuating aural symptoms (hearing, tinnitus or fullness) in the reported ear

C. Not better accounted for by another vestibular diagnosis 
F. Dispenza et al.

Table II. Clinical features and symptoms in the sample.

\begin{tabular}{lccc}
\hline Headache features & Started several years before vertigo spells & Contextually with vertigo spells & Immediately before vertigo spells \\
& $16 / 30$ & $10 / 30$ & $4 / 30$ \\
Accompanying headache & Visual aura & Photophobia & Phonophobia \\
symptoms & $12 / 30$ & $30 / 30$ & $26 / 30$ \\
Hearing symptoms developed & Tinnitus & Fullness & Sensorineural hearing loss \\
during follow-up & $10 / 30$ & $20 / 30$ & $4 / 30$ \\
Other symptoms & Persistent dizziness & Motion sickness & Anxiety \\
& $20 / 30$ & $20 / 30$ & $26 / 30$ \\
\hline
\end{tabular}

tient to fix a point about $30^{\circ}$ over the head with a distance of 1 metre. c-VEMPs were recorded with the negative electrode at the middle third of the sternocleidomastoid muscle ipsilateral to the acoustic stimuli, the positive electrode on the middle third of the clavicle and the ground electrode on the forehead; the patient was lying in a supine position with the head rotate contralateral to the acoustic stimuli and the recording was started by asking the patient to flex the head to the sternum maintaining the position during the examination. The acoustic stimuli were air conducted by earphone inserts as tone burst $500 \mathrm{~Hz}, 120 \mathrm{~dB}$ SPL and repeated 200 times. For each patient, the presence and repeatability of the curve and amplitude and latency of the p10-n10 complex for o-VEMPs and p13-n23 complex for c-VEMPs were evaluated; the asymmetry between two sides over $33 \%$ was considered as pathological ${ }^{8}$.

The electrocochleography (ECochG) was recorded with Socrates, Hedera Biomedics S.r.l. by an extratympanic Lilly TM-Wick electrode as positive, ground electrode on the forehead and the negative electrode on the contralateral mastoid tip. The acoustic stimuli were air conducted by earphone inserts with click stimuli $120 \mathrm{~dB}$ SPL, alternating polarity, rhythm 5,4 msec repeated 2000 times. Both ears were tested. On the curve obtained, the SP/AP ratio was evaluated considering as pathological a result over 0.5 .

The video Head Impulse Test (v-HIT) was done with Eye-SeeCam System Interacoustic. The patient was in a sitting position in front of a point to fix a 1.5 metre distance while the operator casually rotated the head to the right and to the left. The test was considered complete after 20 impulses were accepted. The vestibular-ocular reflex (VOR) gain was considered as pathological if less than 0.8 and if more than $5 \%$ of asymmetry.

The minimum follow-up considered was 24 months since first examination with scheduled revaluation at $6,12,18$ and 24 months; the record was updated in the event that a symptom arose or worsened, or if the instrumental score had modifications. The patients were alerted to call our centre in case of sudden hearing worsening or some modification in their symptoms. Statistical analysis was done with MS Excel performing Chi-squared test and Fisher's exact test. Our IRB approved this clinical study and the work was carried out in accordance with The Code of Ethics of the World Medical Association (Declaration of Helsinki).

\section{Results}

Thirty patients matched inclusion/exclusion criteria and were prospectively followed-up after first examination. The mean age was 45 years (range 18-64 years), with 24 females and 6 males. All patients complained of migraine headache: 16 patients had migraine for years (average 18 years) before vertigo spells, 10 patients had headache contextually to vertigo and in 4 subjects the vertigo spells preceded the headache (average 3 years). Eight patients had family history for vertigo, 6 for migraine alone and 6 for migraine and vertigo.

The visual aura was reported in 12 cases; photophobia was present in all cases, and 26 patients also had phonophobia. Motion sickness was present in 20 cases; anxiety was present in 26 patients and 16 patients reported documented food intolerance. The symptoms referred by patients are summarised in Table II. All patients had normal hearing after first PTA evaluation; 10 patients complained of episodic tinnitus and 20 of transitory fullness sensation, among which 9 had both symptoms. Twenty patients reported dizziness and unsteadiness. All cases had normal gain on v-HIT examination.

In all patients it was possible to record VEMPs. All latency and amplitude values were within the normal range. The finding of VEMPs asymmetry, according to our cut-off of $33 \%$ of difference between sides, resulted in 20 cases, of which 6 with asymmetry of both c-VEMPs and o-VEMPS, 8 with asymmetric c-VEMPs alone and 6 with asymmetric o-VEMPs. Ten patients had normal and symmetric VEMPs response. The asymmetric response more than $33 \%$ resulted in a difference in amplitudes between the two sides, although the values of amplitude were in the normative range: $120 \pm 50 \mu \mathrm{V}$ for $\mathrm{c}-\mathrm{VEMP}$ and $5 \pm 2 \mu \mathrm{V}$ for o-VEMP. In 12 cases, the first ECochG showed a unilateral hydrops because the SP/AP ratio was more than 0.5. The ECochG did not show a significant relationship between fluctuating hearing and SP/AP alteration, and patients with an SP/ AP ratio more than 0.5 at first examination maintained the 
Table III. VEMPs asymmetry evaluation in patients with fluctuating hearing during 2 years of follow-up. Asymmetry of both VEMPs was predictive of fluctuating hearing developed in the side with relatively low amplitude.

\begin{tabular}{lcccc} 
VEMPs asymmetry $>$ 33\% & c-VEMPs & 0-VEMPs & c-VEMPs and o-VEMPs & Total patients \\
Fluctuating hearing & 2 & 4 & 6 & 12 \\
Normal hearing & 4 & 4 & 0 & 8 \\
& $p>0.05$ & $p>0.05$ & $p<0.05$ & 20 \\
\hline
\end{tabular}

same score at further controls. Twelve patients with altered ECochG had VEMPs amplitude and latency in the normal range, but all had both c- and o-VEMPs asymmetry $>33 \%$, with the affected ear showing relatively low amplitude.

During follow-up, the score of instrumental examination (v-HIT, VEMPs and ECochG) of all patients was repeatable and without significant modification. In 16 patients a fluctuation of sensorineural hearing level was documented at PTA and in 4 cases among these the sensorineural hearing loss become stable at an average of $40 \mathrm{~dB}$. In 18 patients re-evaluated for acute vertigo, the spell did not have a noticeable nystagmus at the moment of the visit and repeat instrumental examination did not show any changes.

Statistical analysis was carried out to search for a relationship between fluctuations of hearing and clinical or instrumental features. The presence of asymmetry of both o-VEMPS and c-VEMPs was significantly related to fluctuating hearing $(\mathrm{p}<0.05)$. The data are summarised in Table III. Fluctuating hearing was found in the same side of low amplitude of VEMPs.

\section{Discussion}

This study focused on patients suffering for MV meeting ICDH3-beta criteria. This clinical entity, like migraine headache, is predominant in women (24/30 in our series) in the $3 \mathrm{rd}$ and 4th decade of life. Family predisposition is present in about half of MV patients of our experience, and a possible autosomal dominant genetic transmission may be responsible ${ }^{9}$.

Photophobia and phonophobia are two symptoms frequently described by MV patients, as in our series where they were present in about $90 \%$ of cases ${ }^{10}$. Motion sickness was reported as a frequent symptom in all MV patients by Pagnini et al. ${ }^{11}$; in our findings, $66 \%$ of patients complained of this symptom. The study of vestibular-oculomotor reflex with v-HIT in our series was normal in all subjects with MV, including those who developed fluctuation of hearing during followup. v-HIT applied to MV patients did not show a clinical importance as reported by ElSherif et al. who compared healthy subjects and MV patients and found no alterations in gain or other parameters, underscoring the normality of vestibular-ocular reflex in MV patients ${ }^{12}$.

Often MV patients complain of hearing issues like fullness, tinnitus and even hearing loss. These symptoms associated with migraine symptoms could imply a challenging differential diagnosis with MD ${ }^{1}$. For these reasons, an overlapping syndrome was hypothesised in patients that match both clinical classifications based on referred symptoms ${ }^{6,10}$. Our prospective evaluation of MV patients highlighted that asymmetry of both VEMPs could be a predictor of potential development of an overlapping syndrome with MD.

Several studies have attempted to find a gold standard test that could differentiate VM, MD and non-vestibular migraine ${ }^{13-17}$. The calculation of the SP/AP ratio through electrocochleography (EcochG) has often been reported to be an objective index of endolymphatic hydrops in MD, even though it is limited by low sensitivity and its reliability is strictly dependent on the EcochG technique used, type of stimulus given and stage of MD. In contrast, scarce data are available relative to the use of EcochG in patients with VM. As in the present series of patients, Vitkovic et al. studied 523 patients and found no difference in EcochG test results between VM and MD patients ${ }^{13}$. Lotfi et al. also observed no relevant EcochG differences between patients with VM and healthy subjects in a case series of 20 patients ${ }^{14}$. In contrast, Yollu et al. reported higher SP/AP ratios among patients with VM with respect to migraineurs without complaints of vertigo ${ }^{16}$.

As is known, c-VEMPs is the first instrumental test that shows some alteration in MD due to early involvement of the saccule by endolymphatic hydrops with high sensitivity of the macula to low frequency ${ }^{17,18}$.

In patients with MV, the results of c-VEMPs are conflicting without unique interpretations. Boldingh et al. reported $43 \%$ of their cases had absent potential ${ }^{19}$. Liao and Young evaluated 20 patients with basilar artery migraine with dizziness and c-VEMPs were absent in $35 \%$ of cases ${ }^{20}$.

Zaleski et al. found no difference in c-VEMPs between MV patients and controls, but they reported the absence of oVEMPs in $28 \%$ of cases and low amplitude or asymmetry in large majority of MV patients ${ }^{21}$.

However, alteration of o-VEMPs are variously described in the literature with contradictory results. Makowiec et al. reported that 51 patients among 83 with MV had asymmetry of o-VEMPs, while Zuniga et al. found no difference in amplitude of o-VEMPs between MV patients and normal subjects ${ }^{22,23}$. Hearing in MV patients is in line with general population; on the contrary, in MD hearing loss is a parameter required to achieve a diagnosis ${ }^{4}$. The fluctuating hearing in some of our 
patients during follow-up could indicate a possible correlation between both c-VEMPs and o-VEMPs findings and clinical behaviour of MV over time. The documented fluctuation of hearing associated with vertigo spells lasting hours, which we recorded in some MV patients during follow-up, is more typical for MD compared to MV. In our opinion, some MV patients may develop MD as an evolution of a latent inner ear disease, which for a period of time manifests only with migraine-associated vertigo. Another possible explication could be that MD is latent for a phase of life and manifests with MV-like symptomatology. According to findings of Makowiec et al. ${ }^{23}$, a patient with MV is likely to have abnormal o-VEMPs and normal c-VEMPs and most patients with MD have alterations of c-VEMPs. Although our data are limited by the small number of cases observed, in our series it was noted that a patient with MV symptoms with both o-VEMPs and c-VEMPs asymmetry already has or will develop MD during their lifetime ${ }^{15}$.

\section{Conclusions}

The evolution of MV during the life of patients can have variable progression in which some may develop symptoms typical of MD. The two diseases can be contextually present at the same time, configuring an overlapping syndrome. This condition is an expression of some alterations in the inner ear. The instrumental evaluation of inner ear functions provides information about the status of the system and the asymmetry of both c- and o-VEMPs, although as observed in our small series, seems to be related to a high risk of developing fluctuating hearing during the course of MV. Further study on a larger number of patients could help to better explain the link between MV and MD.

\section{References}

1 Neff BA, Staab JP, Eggers SD, et al. Auditory and vestibular symptoms and chronic subjective dizziness in patients with Meniere's disease, vestibular migraine, and Ménière's disease with concomitant vestibular migraine. Otol Neurotol 2012;33:1235-1244. https://doi. org/10.1097/MAO.0b013e31825d644a

2 Teggi R, Battista RA, Di Berardino F, et al. Evaluation of a large cohort of adult patients with Ménière's disease: bedside and clinical history. Acta Otorhinolaryngol Ital.2020;40:444-449. https://doi. org/10.14639/0392-100X-N0776.

3 Radtke A, Lempert T, Gresty MA, et al. Migraine and Ménière's disease: is there a link? Neurology 2002;59:1700-1704. https://doi. org/10.1212/01.wnl.0000036903.22461.39

4 Headache Classification Committee of the International Headache Society (IHS). The International Classification of Headache Disorders, $3^{\text {rd }}$ edition (beta version). Cephalalgia 2013;33:629-808. https:// doi.org/10.1177/0333102413485658

5 Lopez-Escamez JA, Carey J, Chung WH, et al. Diagnostic criteria for Ménière's disease. J Vestib Res 2015;25:1-7. https://doi.org/10.3233/ ves-150549
6 Murofushi T, Tsubota M, Kitao K, et al. Simultaneous presentation of definite vestibular migraine and definite Ménière's disease: overlapping syndrome of two diseases. Front Neurol 2018;9:749. https://doi. org/10.3389/fneur.2018.00749

7 Nola G, Mostardini C, Salvi C, et al. Validity of Italian adaptation of the Dizziness Handicap Inventory (DHI) and evaluation of the quality of life in patients with acute dizziness. Acta Otorhinolaryngol Ital 2010;30:190-197.

8 Dispenza F, Mazzucco W, Mazzola S, et al. Observational study on risk factors determining residual dizziness after successful benign paroxysmal positional vertigo treatment: the role of subclinical BPPV. Acta Otorhinolaryngologica Ital 2019;39:347-352. https://doi. org/10.14639/0392-100X-2247

9 Bahmad F, DePalma SR, Merchant SN, et al. Locus for familial migrainous vertigo disease maps to chromosome $5 \mathrm{q} 35$. Ann Otol Rhinol Laryngol 2009;118:670-676. https://doi. org/10.1177/000348940911800912

10 Lopez-Escamez JA, Dlugaiczyk J, Jacobs J, et al. Accompanying symptoms overlap during attacks in Ménière's disease and vestibular migraine. Front Neurol 2014;5:265. https://doi.org/10.3389/ fneur.2014.00265

11 Pagnini P, Verrecchia L, Giannoni B, et al. Migraine-related vertigo (MV). Acta Otorhinolaryngol Ital 2003;23:19-27.

12 ElSherif M, Reda MI, Saadallah H, et al. Video head impulse test (vHIT) in migraine dizziness. J Otol 2018;13:65-67. https://doi. org/10.1016/j.joto.2017.12.002.

13 Vitkovic J, Paine M, Rance G. Neuro-otological findings in patients with migraine- and nonmigraine-related dizziness. Audiol Neurootol 2008;13:113-122. https://doi.org/10.1159/000111783

14 Lotfi Y, Mardani N, Rezazade N, et al. Vestibular function in patients with vestibular migraine. Aud Vest Res 2016;25:166-174.

15 Martines F, Dispenza F, Montalbano C, et al. Comparison of electrocochleography and video head impulse test findings in vestibular migraine and Ménière disease: a preliminary study. Int Adv Otol 2020;16:183-189. https://doi.org/10.5152/iao.2020.8165

16 Yollu U, Uluduz DU, Yilmaz M, et al. Vestibular migraine screening in a migraine-diagnosed patient population, and assessment of vestibulocochlear function. Clin Otolaryngol 2017;42:225-233. https:// doi.org/10.1111/coa.12699

17 Maxwell R, Jerin C, Gurkov R. Utilisation of multi-frequency VEMPs improves diagnostic accuracy for Ménière's disease. Eur Arch Otorhinolaryngol 2017;274:85-93. https://doi.org/10.1007/ s00405-016-4206-z

18 Scarpa A, Gioacchini FM, Cassandro E, et al. Clinical application of cVEMPs and oVEMPs in patients affected by Ménière's disease, vestibular neuritis and benign paroxysmal positional vertigo: a systematic review. Acta Otorhinolaryngol Ital 2019;39:298-307. https://doi. org/10.14639/0392-100X-2104

19 Boldingh MI, Ljostad U, Mygland A, et al. Vestibular sensitivity in vestibular migraine: VEMPs and motion sickness susceptibility. Cephalalgia 2011;31:1211-1219. https://doi.org/10.1177/0333102411409074

20 Liao LJ, Young YH. Vestibular evoked myogenic potentials in basilar artery migraine. Laryngoscope 2004;114:1305-1309. https://doi. org/10.1097/00005537-200407000-00031

21 Zaleski A, Bogle J, Starling A, et al. Vestibular evoked myogenic potentials in patients with vestibular migraine. Otol Neurotol 2015;36:295-302. https://doi.org/10.1097/mao.0000000000000665

22 Zuniga MG, Janky KL, Schubert MC, et al. Can vestibular-evoked myogenic potentials help differentiate Ménière disease from vestibular migraine? Otolaryngol Head Neck Surg 2012;146:788-796. https://doi.org/10.1177/0194599811434073

23 Makowiec KF, Piker EG, Jacobson GP, et al. Ocular and cervical vestibular evoked myogenic potentials in patients with vestibular migraine. Otol Neurotol 2018;39:e561-e567. https://doi.org/10.1097/ mao.0000000000001880 